\title{
GRADED TRANSCENDENTAL EXTENSIONS OF GRADED FIELDS
}

\section{BOULAGOUAZ}

Received 2 October 2002

We study transcendency properties for graded field extension and give an application to valued field extensions.

2000 Mathematics Subject Classification: 12F20, 16W50.

1. Introduction. An important tool to study rings with valuation is the socalled associated graded ring construction: to a valuation ring $R$, we can associate a ring $\operatorname{gr}(R)$ graded by the valuation group. This ring is often easier to study, and one tries to lift properties back from $\operatorname{gr}(R)$ to $R$. This principle has been recently applied to rings of differential operators (see [9]), the Brauer group (see, e.g., [8]), and to valuations on division algebras (see [1, 11, 12]). This has been one of the motivations to study graded rings, see [10] for a detailed discussion. In a sense, the easiest example of a graded ring is a graded field, this is a commutative graded ring in which every homogeneous element is invertible, and the terminology has been introduced in [13].

This note is a continuation of earlier work of the author (see [3, 4, 5, 6]), in which graded fields and graded division rings are studied with special emphasis on applications to valuation theory. The aim of this note is to introduce and study the notion of gr-transcendental graded field extension, at least in the case where the grading group is torsion-free abelian; application to valued field extensions leads to three different notions of transcendental extensions of valued fields.

In Section 2, we recall some basic results on graded ring theory and on gradings on polynomial rings. We introduce the notions of gr-algebraically freeness and gr-transcendental extension in Section 3 and prove some elementary properties (see, e.g., Proposition 3.4). In Section 4, we look at two special cases: unramified graded field extensions, where the grading groups of both graded fields are the same, and totally ramified extensions, where the parts of degree zero of both extensions coincide. The transcendency can be described explicitly in both cases; combination of the two situations leads to the existence of a gr-transcendency basis in general (Proposition 4.5) and to the notion of gr-transcendency degree. In Section 5, we give a structure theorem for purely gr-transcendental graded field extensions of divisible type (Proposition 5.1); 
a graded field extension is of divisible type if the quotient of the two grading groups is torsion. In Section 6, we apply our results to valued fields and introduce the notions of gradually, residually, and valuatively transcendental valued field extensions.

\section{Preliminaries}

2.1. Graded rings. Let $\Gamma$ be a torsion-free abelian group and $R$ a commutative ring. All rings considered in this note will be commutative. Recall that $R$ is called a $\Gamma$-graded ring if $R=\oplus_{\delta \in \Gamma} R_{\delta}$, where $R_{\delta}$ is an additive subgroup of $R$, such that $R_{\delta} R_{\gamma} \subset R_{\delta+\gamma}$, for all $\gamma, \delta \in \Gamma$. We say that $a \neq 0 \in R_{\delta}$ is homogeneous of degree $\delta$, and we then write $\operatorname{deg}(a)=\delta$. Let $H(R)=\left(\cup_{\delta \in \Gamma} R_{\delta}\right) \backslash\{0\}$ be the set of all homogeneous elements of $R$. If $a=\sum_{\delta \in G} a_{\delta}$ with $a_{\delta} \in R_{\delta}$, then $a_{\delta}$ is called the homogeneous component of $a$ of degree $\delta ; \Gamma_{R}=\left\{\lambda \in \Gamma \mid R_{\lambda} \neq\{0\}\right\}$ is called the support of the graded ring $R$ and $R$ is a domain if and only if $H(R)$ has no zero divisors; in this case $\Gamma_{R}$ is a submonoid of $\Gamma$. We will say that $R$ is a graded ring of type $\Gamma$ if $R$ is $\Gamma$-graded with $\Gamma_{R}=\Gamma$. A $\Gamma$-graded commutative ring $R$ is called a graded field if every nonzero homogeneous element of $R$ is invertible. If $R$ is a graded field, then $\Gamma_{R}$ is a subgroup of $\Gamma$ and is called the grading group of $R$. The rational closure of $\Gamma_{R}$ is then denoted by $\Delta_{R}=\Gamma_{R} \otimes_{\mathbb{Z}} \mathbb{Q}$. In this case, $H(R)$ is a group, called the group of homogeneous elements of $R$.

If $R$ and $S$ are $\Gamma$-graded rings, then $f: R \rightarrow S$ is called a homomorphism of graded rings if $f$ is grade preserving, that is, $f\left(R_{\tau}\right) \subset S_{\tau}$ for all $\tau \in \Gamma$.

2.2. Gradings on polynomial rings. Throughout, $\Gamma$ will be a torsion-free abelian group. Let $R$ be a $\Gamma$-graded commutative ring and assume that $H(R)$ contains no zero divisors. Localizing $R$ at the multiplicatively closed set $H(R)$, we obtain a $\Gamma$-graded field $\operatorname{Fr}_{\mathrm{gr}}(R)$, called the graded field of fractions of $R$. The support of $\operatorname{Fr}_{\mathrm{gr}}(R)$ is the subgroup $\left\{\alpha-\beta \mid \alpha, \beta \in \Gamma_{R}\right\}$ of $\Gamma$.

Let $X=\left\{X_{i} \mid i \in I\right\}$ be a (finite or infinite) set of variables and $\Delta_{\Gamma}=\Gamma \otimes_{\mathbb{Z}} \mathbb{Q}$ the divisible closure of $\Gamma$. Consider a map $\omega: X \rightarrow \Delta_{\Gamma}$ and write $\omega\left(X_{i}\right)=\delta_{i}$. Then $\omega$ defines a $\Delta_{\Gamma}$-grading on $R[X]$ by taking $\operatorname{gr}\left(X_{i}\right)=\delta_{i}$. This graded ring is denoted by $R[X]^{\omega}$. Clearly, the support of $R[X]^{\omega}$ is contained in $\Gamma_{R}[\omega(X)]$, the submonoid of $\Delta_{\Gamma}$ generated by $\Gamma_{R}$ and $\omega(X)$.

We call $P \in R[X]$ homogenizable if there exists $\omega: X \rightarrow \Delta_{\Gamma}$ such that $P \in$ $H\left(R[X]^{\omega}\right)$.

Now assume that $R$ is a graded field. Then $R[X]^{\omega}$ is a domain, and we can consider $\operatorname{Fr}_{\mathrm{gr}}\left(R[X]^{\omega}\right)=R(X)^{\omega}$, called the $\omega$-graded field of fractions of $R[X]$.

Let $R$ be a graded subring of a $\Gamma$-graded field $S$, and $A \subset H(S)$. Then the subring $R[A]$ of $S$ generated by $R$ and $A$ is graded. Its graded field of fractions $R(A)$ is the smallest graded subfield of $S$ containing $R$ and $A$. If $B \subset H(S)$ is another subset, then $R[A \cup B]=R[A][B]$ and $R(A \cup B)=R(A)(B)$. 
3. Graded transcendental extensions of graded fields. Let $\Gamma$ be a torsionfree abelian group and $R \subset S$ an extension of $\Gamma$-graded fields. For $T=\left\{t_{1}, \ldots, t_{s}\right\} \subset$ $H(S)$, we consider the map $\omega_{T}:\left\{X_{1}, \ldots, X_{s}\right\} \rightarrow \Gamma, \omega_{T}\left(X_{i}\right)=\operatorname{deg}\left(t_{i}\right)$.

(1) $T$ is called gr-algebraically free over $R$ if there are no nonzero $P \in$ $H\left(R\left[X_{1}, \ldots, X_{S}\right]^{\omega_{T}}\right)$ such that $P\left(t_{1}, \ldots, t_{s}\right)=0$;

(2) $L \subset H(S)$ is called gr-algebraically free over $R$ if every finite subset of $L$ is gr-algebraically free;

(3) $T$ is called a gr-transcendency basis of $S$ over $R$ if $T$ is a maximal gralgebraically free subset of $H(S)$ (for the inclusion);

(4) $a \in H(S)$ is called gr-transcendental over $R$ if $\{a\}$ is gr-algebraically free; otherwise $a$ is called gr-algebraic;

(5) $R \subset S$ is called a gr-algebraic graded field extension if every $a \in H(S)$ is gr-algebraic; otherwise $R \subset S$ is called a transcendental graded field extension.

Every graded field extension of finite degree is gr-algebraic (see [6] or [7]); consequently, $[S: R]=\infty$ if $R \subset S$ is transcendental.

If $T=\left\{t_{1}, \ldots, t_{s}\right\} \subset H(S)$ is gr-algebraically free, then every $t_{i}$ is gr-transcendental over $R$ and $T \cap H(R)=\varnothing$.

Proposition 3.1. Let $S / R$ be a graded field extension and let $T \subset H(S)$. The set $T$ is a gr-transcendency basis of $S$ over $R$ if and only if $S / R(T)$ is gr-algebraic and $T$ is gr-algebraically free over $R$.

Proof. First, Assume that $T$ is a gr-transcendency basis and take $a \in H(S) \backslash$ $T$. Then $T \cup\{a\}$ is not gr-algebraically free, so there exists $T^{\prime}=\left\{t_{1}, \ldots, t_{s}, a\right\} \subset$ $T \cup\{a\}$ and $0 \neq f\left(X_{1}, \ldots, X_{s}, Y\right) \in R\left[X_{1}, \ldots, X_{s}, Y\right]^{\omega_{T^{\prime}}}$ such that $f\left(t_{1}, \ldots, t_{s}, a\right)=$ 0 . We can write

$$
f\left(X_{1}, \ldots, X_{S}, Y\right)=\sum_{i=1}^{r} f_{i}\left(X_{1}, \ldots, X_{S}\right) Y^{i}
$$

with at least one $f_{i} \neq 0$. It follows that

$$
\sum_{i=1}^{r} f_{i}\left(t_{1}, \ldots, t_{s}\right) a^{i}=0
$$

and $a$ is gr-algebraic over $R(T)$.

Conversely, let $T \subset H(S)$ be gr-algebraically free and assume that $S / R(T)$ is gr-algebraic. For $x \in H(S)$, there exists $f(X)=\sum_{i=1}^{r} a_{i} X^{i} \in H\left(R(T)[X]^{(\operatorname{gr}(x))}\right)$ such that $f(x)=0$. We can find $T^{\prime}=\left\{t_{1}, \ldots, t_{s}\right\} \subset T$ such that each $a_{i}$ can be written in the form

$$
a_{i}=\frac{N_{i}\left(t_{1}, \ldots, t_{s}\right)}{D\left(t_{1}, \ldots, t_{s}\right)}
$$


with $N_{i}, D \in H\left(R\left[X_{1}, \ldots, X_{s}\right]^{\omega_{T^{\prime}}}\right)$ and $d=D\left(t_{1}, \ldots, t_{s}\right) \neq 0$. The polynomial

$$
P\left(X_{1}, \ldots, X_{s}, X\right)=\sum_{i=1}^{r} N_{i}\left(T_{1}, \ldots, T_{s}\right) X^{i}
$$

is homogenizable and $P\left(t_{1}, \ldots, t_{s}, x\right)=0$. Hence, $T \cup\{x\}$ is not gr-algebraically free.

We call $S$ a pure gr-transcendental graded field extension of $R$ if there exists a (possibly empty) gr-transcendency basis $T$ of $R$ such that $S=R(T)$. Such a basis is called a generating gr-transcendency base.

REMARK 3.2. Let $A=\left\{a_{i} \mid i \in I\right\} \subset H(S)$ and consider $\omega_{A}: X=\left\{X_{i} \mid i \in\right.$ $I\} \rightarrow \Gamma, \omega\left(X_{i}\right)=\operatorname{deg}\left(a_{i}\right)$. Then we have a canonical surjection of graded rings $\phi_{A}: R[X]^{\omega_{A}} \rightarrow R[A], \phi_{A}\left(X_{i}\right)=a_{i}$. The set $A$ is gr-algebraically free if and only if $\phi_{A}$ is an isomorphism of graded rings. The map $\phi_{A}$ induces a morphism of graded fields

$$
\psi_{A}: \operatorname{Fr}_{\mathrm{gr}}\left(R[X]^{\omega_{A}}\right)=R(X)^{\omega_{A}} \longrightarrow S
$$

and $A$ is a generating gr-transcendency base of $S$ over $R$ if and only if $\psi_{A}$ is an isomorphism.

Proposition 3.3. Let $S / R$ be a $\Gamma$-graded field extension and $T \subset H(S)$. Assume that $T$ is the disjoint union of two subsets $L$ and $C$.

(1) The subset $C$ is gr-algebraically free over $R(L)$.

(2) Every $h \in H(R(T)) \backslash R$ is gr-transcendental over $R$.

Proof. (1) Assume that $C$ is not gr-algebraically free over $R(L)$. Then there exist finite subsets $L_{1}=\left\{t_{1}, \ldots, t_{s}\right\} \subset L$ and $C_{1}=\left\{t_{s+1}, \ldots, t_{r}\right\} \subset C$ and a homogeneous polynomial $P \in R\left(t_{1}, \ldots, t_{s}\right)\left[X_{s+1}, \ldots, X_{r}\right]^{\omega_{C_{1}}}$ such that $P\left(t_{s+1}, \ldots, t_{r}\right)=$ 0 . The coefficients of $P$ are quotients of homogeneous polynomials in $t_{1}, \ldots, t_{s}$. Let $Q$ be a common multiple of the denominators. Then we can write

$$
P=\frac{F\left(t_{1}, \ldots, t_{s}, X_{s+1}, \ldots, X_{r}\right)}{Q\left(t_{1}, \ldots, t_{s}\right)}
$$

with $F \in H\left(R\left[X_{1}, \ldots, X_{s}, X_{s+1}, \ldots, X_{r}\right]^{\omega_{L_{1}} \cup \omega_{C_{1}}}\right)$. Now,

$$
F\left(t_{1}, \ldots, t_{s}, t_{s+1}, \ldots, t_{r}\right)=0,
$$

contradicting the fact that $T$ is gr-algebraically free over $R$.

(2) For $h \in H(R(T))$, we have a finite subset $T_{1}=\left\{t_{1}, \ldots, t_{r}\right\}$ of $T$ such that $h \in H\left(R\left(T_{1}\right)\right)$, and we can write

$$
h=\frac{P\left(t_{1}, \ldots, t_{r}\right)}{Q\left(t_{1}, \ldots, t_{r}\right)}
$$

with $P, Q \in H\left(R\left[X_{1}, \ldots, X_{r}\right]^{\omega_{T_{1}}}\right)$ and $Q\left(t_{1}, \ldots, t_{r}\right) \neq 0$. 
Assume that $h$ is gr-algebraic over $R$. Then there exists $F \in H\left(R[X]^{\omega_{\{h\}}}\right)$ such that $F(h)=0$. Write $F(X)=\sum_{i=0}^{n} a_{i} X^{i}$. Then,

$$
Q\left(t_{1}, \ldots, t_{r}\right)^{n} F(h)=\sum_{i=0}^{n} a_{i} Q\left(t_{1}, \ldots, t_{r}\right)^{n-i} P\left(t_{1}, \ldots, t_{r}\right)^{i}=0 .
$$

Now,

$$
F\left(X_{1}, \ldots, X_{r}\right)=\sum_{i=0}^{n} a_{i} Q\left(X_{1}, \ldots, X_{r}\right)^{n-i} P\left(X_{1}, \ldots, X_{r}\right)^{i}
$$

is a homogeneous polynomial in $R\left[X_{1}, \ldots, X_{r}\right]^{\omega_{T_{1}}}$ and $F\left(t_{1}, \ldots, t_{r}\right)=0$. This contradicts the fact that $T$ is gr-algebraically free.

Proposition 3.4. Let $Q / S$ and $S / R$ be two $\Gamma$-graded field extensions and let $T_{Q}$ be a gr-algebraically free subset of $Q$ over $S$ and $T_{S}$ a gr-algebraically free subset of $S$ over $R$. Then the following properties hold:

(1) $T_{Q} \cap T_{S}=\varnothing$;

(2) $T_{Q} \cup T_{S}$ is a gr-algebraically free subset of $Q$ over $R$;

(3) $T_{Q} \cup T_{S}$ is a gr-transcendency base of $Q$ over $R$ if and only if $T_{Q}$ and $T_{S}$ are gr-transcendency bases, respectively of $Q$ over $S$ and of $S$ over $R$;

(4) $Q / R$ is a pure gr-transcendental extension if and only if $Q / S$ and $S / R$ are pure gr-transcendental extensions.

Proof. (1) The proof follows from the fact that $T_{Q} \cap H(S)=\varnothing$ and $T_{S} \subset$ $H(S)$.

(2) Clearly, finite subsets

$$
L_{1}=\left\{t_{1}, \ldots, t_{s}\right\} \subset T_{Q}, \quad L_{2}=\left\{t_{s+1}, \ldots, t_{s+r}\right\} \subset T_{S}
$$

are gr-algebraically free over $R$. Assume that $L=L_{1} \cup L_{2}$ is not gr-algebraically free over $R$. Then $L_{1} \neq \varnothing$ and $L_{2} \neq \varnothing$, and there exists a nonzero homogenizable $f \in R\left[X_{1}, \ldots, X_{s+r}\right]$ such that $f\left(t_{1}, \ldots, t_{s+r}\right)=0$. Then $g=f\left(X_{1}, \ldots, X_{s}\right.$, $\left.t_{s+1}, \ldots, t_{s+r}\right)$ is a homogeneous polynomial in $S\left[X_{1}, \ldots, X_{s}\right]$, and $g\left(t_{1}, \ldots, t_{s}\right)=$ 0 , hence $Q$ is not gr-algebraically free over $S$, which is a contradiction.

(3) Let $T_{Q}$ and $T_{S}$ be gr-transcendency bases, respectively of $Q$ over $S$ and of $S$ over $R$, and consider free $L \subset H(Q)$ gr-algebraically over $R$ and strictly containing $T_{Q} \cup T_{S}$. Then

$$
L=(L \cap(Q \backslash S)) \cup(L \cap S) .
$$

We have that at least one of the two inclusions $L \cap(Q \backslash S) \subset T_{Q}$ and $L \cap S \subset T_{S}$ is strict, and $L \cap(Q \backslash S) \subset H(Q)$ and $L \cap S \subset H(S)$ are gr-algebraically free over, respectively, $S$ and $R$. This contradicts the hypothesis.

Conversely, assume that $T_{Q} \cup T_{S}$ is a gr-transcendency base of $Q$ over $R$ and that $T_{Q}$ is not a maximal gr-algebraically free part of $H(Q)$ over $S$. Let $L$ be a 
gr-algebraically free part of $H(Q)$ over $S$ strictly containing $T_{Q}$. Then $L \cup T_{S}$ strictly contains $T_{Q} \cup T_{S}$ and is gr-algebraically free over $R$ by part (2), which is a contradiction. We use the same argument if $T_{S}$ is not maximal.

(4) If $S=R\left(T_{S}\right)$ and $Q=S\left(T_{Q}\right)$, then $Q=S\left(T_{Q}\right)=R\left(T_{S}\right)\left(T_{Q}\right)=R\left(S_{T} \cup T_{Q}\right)$, and it follows from part (2) that $S_{T} \cup T_{Q} \subset H(Q)$ is gr-algebraically free over $R$. Conversely, let $Q=R(L)$ with $L \subset H(Q)$ gr-algebraically free over $R$. Let $L_{S}=L \cap H(S)$ and $L_{Q}=L \backslash L_{S}$. It follows from part (3) that $L_{S}$ and $L_{Q}$ are grtranscendency bases of $S$ over $R$ and of $Q$ over $S$. If there exists $x \in S \backslash R\left(L_{S}\right)$, then $x$ is gr-algebraic over $R\left(L_{S}\right)$. But it follows from part (2) of Proposition 3.3 that every $x \in Q \backslash R\left(L_{S}\right)$ is gr-transcendental over $R\left(L_{S}\right)$, so we have a contradiction.

Finally, $Q=R(L)=R\left(L_{Q} \cup L_{S}\right)=R\left(L_{S}\right)\left(L_{Q}\right)=S\left(L_{Q}\right)$.

4. Unramified and totally ramified graded field extensions. We call an extension $S / R$ of $\Gamma$-graded fields unramified if $\Gamma_{S}=\Gamma_{R}$.

Proposition 4.1. Let $S / R$ be an unramified $\Gamma$-graded field extension.

(1) Every transcendency basis $T$ of $S_{0} / R_{0}$ is a gr-transcendency basis of $S / R$.

(2) If $T$ is a gr-transcendency basis of $S / R$, then for each $t \in T$, there exists $r_{t} \in H(R)$ such that $T_{0}=\left\{t / r_{t} \mid t \in T\right\}$ is a transcendency basis of $S_{0} / R_{0}$.

Proof. (1) If $T$ is not gr-algebraically free over $R$, then there exist $\left\{t_{1}, \ldots\right.$, $\left.t_{s}\right\} \subset T \subset R_{0}$ and $P \in H\left(R\left[X_{1}, \ldots, X_{S}\right]^{\omega_{T}}\right)$ such that

$$
P\left(t_{1}, \ldots, t_{s}\right)=0 \text {. }
$$

In $R\left[X_{1}, \ldots, X_{S}\right]^{\omega_{T}}$, we have that $\operatorname{deg}\left(X_{i}\right)=0$, for all $i$, and we can conclude that all the coefficients of $P$ are homogeneous of the same degree $\delta$ (which is also the degree of $P$ ). Take $x \neq 0 \in R_{\delta}$, then $x^{-1} P\left(t_{1}, \ldots, t_{s}\right)=0$, and $x^{-1} P \in$ $R_{0}\left[X_{1}, \ldots, X_{s}\right]$, hence $T$ is not algebraically free over $R_{0}$, which is a contradiction. From the fact that $S_{0} / R(T)_{0}$ is algebraic and $\Gamma_{S}=\Gamma_{R}=\Gamma_{R(T)}$ is a torsion group over $\Gamma_{R(T)}$, we conclude that $S / R(T)$ is gr-algebraic (see [6, Proposition 1 , page 24]).

(2) Let $T$ be a gr-transcendency basis of $S / R$. For every $t \in T$, we choose $r_{t} \in R$ such that $\operatorname{deg}\left(r_{t}\right)=\operatorname{deg}(t)$ (using the fact that $\Gamma_{R}=\Gamma_{S}$ ). Then $L_{T}=$ $\left\{t / r_{t} \mid t \in T\right\} \subset S_{0}$ is still a gr-transcendency basis of $S / R$. From the fact that $L_{T}$ is gr-algebraically free, it follows immediately that $L_{T}$ is algebraically free; also the fact that $S / R\left(L_{T}\right)$ is gr-algebraic entails that $S_{/}\left(R\left(L_{T}\right)_{0}\right)$ is algebraic, and the proof is finished after we remark that $R\left(L_{T}\right)_{0}=R_{0}\left(L_{T}\right)$.

COROLLARY 4.2. Every unramified graded field extension $S / R$ has a grtranscendency basis and all the gr-transcendency bases have the same cardinality, equal to the transcendency degree of $S_{0} / R_{0}$.

We call an extension $S / R$ of $\Gamma$-graded fields totally ramified if $R_{0}=S_{0}$. 
Proposition 4.3. If $S / R$ is a totally ramified extension of $\Gamma$-graded fields, then $T \subset H(S)$ is gr-algebraically free over $R$ if and only if $\Gamma_{T}=\{\operatorname{deg}(t) \mid t \in T\}$ is linearly free in $\Gamma_{S} / \Gamma_{R}$.

Proof. Assume first that $T$ is not algebraically free. Then there exist $T^{\prime}=$ $\left\{t_{1}, \ldots, t_{s}\right\} \subset T$ and a nonzero $P \in H\left(R\left[X_{1}, \ldots, X_{s}\right]^{\omega_{T}}\right)$ such that $P\left(t_{1}, \ldots, t_{s}\right)=0$. The polynomial $P$ can be written as a sum of monomials, and at least two of them are different from 0 , say

$$
a X_{1}^{n_{1}} \cdots X_{s} n_{s}, \quad b X_{1}^{m_{1}} \cdots X_{s} m_{s} .
$$

All these monomials have the same degree, hence

$$
\begin{gathered}
\operatorname{deg}(a)+\sum_{j=1}^{s} n_{j} \operatorname{deg}\left(t_{j}\right)=\operatorname{deg}(b)+\sum_{j=1}^{s} m_{j} \operatorname{deg}\left(t_{j}\right), \\
\sum_{j=1}^{s}\left(n_{j}-m_{j}\right) \operatorname{deg}\left(t_{j}\right)=\operatorname{deg}(b)-\operatorname{deg}(a) \in \Gamma_{R},
\end{gathered}
$$

so $\Gamma_{T}$ is not linearly free modulo $\Gamma_{R}$.

Conversely, assume that there exists $T^{\prime}=\left\{t_{1}, \ldots, t_{s}\right\} \subset T$ such that $\Gamma_{T^{\prime}}$ is not linearly free modulo $\Gamma_{R}$. Then there exist $l_{1}, \ldots, l_{s} \in \mathbb{Z}$ such that

$$
\sum_{k=1}^{s} l_{k} \operatorname{deg}\left(t_{k}\right)=\lambda \in \Gamma_{R} .
$$

Take $a \neq 0 \in H(R)$ such that $\operatorname{deg}(a)=\lambda$. Then,

$$
b=a^{-1} t_{1}^{l_{1}} \cdots t_{s}^{l_{s}} \in R_{0} .
$$

For every $m \in\{1, \ldots, s\}$, we take

$$
\begin{aligned}
& i_{m}=l_{m}, \quad j_{m}=0 \quad \text { if } l_{m} \geq 0, \\
& i_{m}=0, \quad j_{m}=-l_{m} \quad \text { if } l_{m}<0 .
\end{aligned}
$$

The polynomial

$$
P\left(X_{1}, \ldots, X_{s}\right)=a^{-1} b^{-1} X_{1}^{i_{1}} \cdots X_{s} i_{s}-X_{1}^{j_{1}} \cdots X_{s} j_{s}
$$

is homogeneous in $R\left[X_{1}, \ldots, X_{s}\right]^{\omega_{T^{\prime}}}$, and $P\left(t_{1}, \ldots, t_{s}\right)=0$, so it follows that $T^{\prime}$ is not gr-algebraically free.

COROLLARY 4.4. Every totally ramified graded field extension $S / R$ has a grtranscendency basis and all the gr-transcendency bases have the same cardinality, equal to the rank of the abelian group $\Gamma_{S} / \Gamma_{R}$.

Proof. Take a maximal free subgroup $F$ of $\Gamma_{S} / \Gamma_{R}$; then $\Gamma_{S} / \Gamma_{R}(F)$ is torsion. For every $f \in F$, choose $t_{f} \in H(S)$ such that $\operatorname{deg}\left(t_{f}\right)$ represents $f$ in $\Gamma_{S} / \Gamma_{R}$. 
It follows from Proposition 4.3 that $T=\left\{t_{f} \mid f \in F\right\}$ is gr-algebraically free. Finally, $\Gamma_{R(T)}=\Gamma_{R}\left(\Gamma_{T}\right)$ and $R(T)_{0}=R_{0}$, so $S_{0} / R(T)_{0}=R_{0} / R_{0}$ is algebraic. It follows that $S / R(T)$ is gr-algebraic if and only if $\left(\Gamma_{S} / \Gamma_{R}\left(\Gamma_{T}\right)=\Gamma_{S}\right) / \Gamma_{R}(F)$ is torsion, see [6, Proposition 1, page 24].

We now look at the general case: if $S / R$ is an extension of $\Gamma$-graded fields, then $S / R\left(S_{0}\right)$ is a totally ramified extension and $R\left(S_{0}\right) / R$ is an unramified extension. The above results show that $S / R$ has a gr-transcendency basis with cardinality equal to the sum of the transcendency degree of $S_{0} / R_{0}$ and the rank of $\Gamma_{S} / \Gamma_{R}$. Moreover, we have the following result.

Proposition 4.5. Let $S / R$ be a $\Gamma$-graded field extension. Then all gr-transcendency bases of $S / R$ have the same cardinality, equal to the sum of the transcendency degree of $S_{0} / R_{0}$ and the rank of $\Gamma_{S} / \Gamma_{R}$.

Proof. Let $T$ be a gr-transcendency basis of $S / R$. Then $\Gamma_{R(T)}=\Gamma_{R}\left(\Gamma_{T}\right)$, and $\Gamma_{S} / \Gamma_{R(T)}$ is torsion since $S / R(T)$ is gr-algebraic. Applying Zorn's lemma to the set $\mathcal{T}$ consisting of $T^{*} \subset T$ such that $\Gamma_{T *}$ is linearly free over $\Gamma_{R}$, and such that two different elements in $T^{*}$ have different degrees, we obtain a maximal subset $T_{m}$ satisfying these two properties. Then $\Gamma_{R}\left(\Gamma_{T}\right) / \Gamma_{R}\left(\Gamma_{T_{m}}\right)$ and, a fortiori, $\Gamma_{S} / \Gamma_{R}\left(\Gamma_{T_{m}}\right)$ are torsion, and $T_{m}$ is a basis of $\Gamma_{S} / \Gamma_{R}$, proving that $T_{m}$ is a grtranscendency basis of $S / R\left(S_{0}\right)$.

On the other hand, the map $\operatorname{deg}: H(S) \rightarrow \Gamma_{S}$ is a group homomorphism and its kernel $S_{0}^{*}$ is a multiplicative subgroup of $S_{0}$. The image of $H\left(R\left(T_{m}\right)\right)$ under deg is $\Gamma_{R}\left(\Gamma_{T_{m}}\right)$ and the inverse image of $\Gamma_{R}\left(\Gamma_{T_{m}}\right)$ is $H\left(R\left(T_{m}\right)\right) S_{0}^{*}$.

For every $t \in \bar{T}=T \backslash T_{m}$, there exists $n_{t} \in \mathbb{N}$ such that $n_{t} \operatorname{deg}(t) \in \Gamma_{R}\left(\Gamma_{T_{m}}\right)$ or, equivalently, $t^{n_{t}} \in H\left(R\left(T_{m}\right)\right) S_{0}^{*}$. Therefore,

$$
t^{n_{t}} H\left(R\left(T_{m}\right)\right) \cap S_{0}^{*} \neq \varnothing
$$

Let $G$ be the multiplicative subgroup of $H(R)(T)$ generated by $H(R)$ and $T$ in $H(S)$. Then an element $x \in R(T) \cap S_{0}$ can be written as a quotient $\sum_{i} a_{i}$ by $\sum_{j} b_{j}$, where the $a_{i}$ and $b_{j}$ are elements of $G \cap S_{0}$. Hence,

$$
R(T) \cap S_{0}=R_{0}(G) \cap S_{0}
$$

and $S_{0} / R_{0}\left(G \cap S_{0}\right)$ is algebraic.

For every $t \in \bar{T}$, we choose $\tilde{t} \in t^{n_{t}} H\left(R\left(T_{m}\right)\right) \cap S_{0}$ and we put

$$
\tilde{T}=\{\tilde{t} \mid t \in T\}
$$

and $\tilde{G}=R_{0}^{*}(\tilde{T})$, the subgroup of $S_{0}^{*}$ generated by $R_{0}^{*}$ and $\tilde{T}$. Then $\left(G \cap S_{0}\right) / \tilde{G}$ is torsion, hence $R(T) \cap S_{0}$ is algebraic over $R_{0}(\tilde{G})=R_{0}(\tilde{T})$. Now $S_{0} / R(T) \cap S_{0}$ is algebraic, so $S_{0} / R_{0}(\tilde{T})$ is also algebraic. We know that $\tilde{T}$ is gr-algebraically 
free over $R_{0}$, so $\tilde{T}$ is a gr-transcendency basis of $R\left(S_{0}\right) / R$ and $T_{m} \cup \tilde{T}$ is a grtranscendency basis of $S / R$. To finish the proof, it suffices to remark that the map $T \rightarrow T_{m} \cup \tilde{T}$, mapping $t$ to $t$ if $t \in T_{m}$, and to $\tilde{t}$ otherwise, is a bijection.

The cardinality of a gr-transcendency basis of $S / R$ is called the gr-transcendency degree of $S / R$, and is denoted by $[S: R]_{t}$.

5. Extensions of divisible type. We say that an extension $S / R$ of $\Gamma$-graded fields is of divisible type if $\Gamma_{S} / \Gamma_{R}$ is a torsion group. In this situation, we have that $\Gamma_{S} \subset \Delta_{R}$.

Proposition 5.1. Let $R$ be a $\Gamma$-graded field $X=\left\{X_{i} \mid i \in I\right\}$ a set of variables, and $\omega: X \rightarrow \Delta_{R}$.

(1) The graded field extension $R(X)^{\omega} / R$ is pure gr-transcendental of divisible type; $X$ is a generating gr-transcendency basis.

(2) Every pure gr-transcendental graded field extension of divisible type of $R$ is gr-isomorphic to $R(X)^{\omega}$ for a suitable choice of $X$ and $\omega$.

Proof. (1) It is clear that $R(X)^{\omega} / R$ is an extension of divisible type since $\Gamma_{R(X) \omega} \subset \Delta_{R}$ and $\Delta_{R} / \Gamma_{R}$ is torsion. If $X$ is not gr-algebraically free over $R$, then there exist $F=\left\{X_{1}, \ldots, X_{r}\right\} \subset X$ finite and a polynomial $P \in H\left(R\left[Y_{1}, \ldots\right.\right.$, $\left.\left.Y_{r}\right]^{\omega_{|F|}}\right)$ such that $P\left(X_{1}, \ldots, X_{r}\right)=0$. But then $P$ is the zero polynomial.

(2) Let $S / R$ be a pure gr-transcendental graded field extension of divisible type with gr-transcendency basis $T=\left\{t_{i} \mid i \in I\right\}$. Then $\operatorname{deg}\left(t_{i}\right) \in \Delta_{R}$. Let $X=$ $\left\{X_{i} \mid i \in I\right\}$ be a set of indeterminates and consider $\omega: X \rightarrow \Delta_{R}, \omega\left(X_{i}\right)=$ $\operatorname{deg}\left(t_{i}\right)$. By Remark 3.2, the map $\psi: R(X) \omega \rightarrow S$ defined by $\psi\left(X_{i}\right)=t_{i}$ is an isomorphism of graded fields.

Proposition 5.2. Let $R$ be a $\Gamma$-graded field and $\omega: X=\left\{X_{i} \mid i \in I\right\} \rightarrow \Delta_{R}$. Then $\left(R(X)^{\omega}\right)_{0} / R_{0}$ is a pure transcendental field extension and $\left[\left(R(X)^{\omega}\right)_{0}\right.$ : $\left.R_{0}\right]_{t}=\left[R(X)^{\omega}: R\right]_{t}$.

Proof. Let $\mathscr{L}$ be the set consisting of all couples $\left(Y, B_{Y}\right)$ with $Y \subset X$ and $\left(R(Y)^{\omega_{\mid Y}}\right)_{0} / R_{0}$ a pure transcendental field extension with generating transcendency basis $B_{Y}$. The set $\mathscr{L}$ is partially ordered: $\left(Y, B_{Y}\right) \leq\left(Z, B_{Z}\right)$ if and only if $Y \subset Z$ and $B_{Y} \subset B_{Z}$.

Take $\left(Y, B_{Y}\right) \in \mathscr{L}$ and $Y \subset Z \subset X$. Remark that there exists $B_{Z} \subset R(Z)_{0}^{\omega_{\mid Z}}$ such that $B_{Y} \subset B_{Z}$ and $\left(Z, B_{Z}\right) \in \mathscr{L}$ if and only if $R(Z)_{0}^{\omega_{\mid Z}} / R(Y)_{0}^{\omega_{\mid Y}}$ is a purely transcendental field extension. Indeed, if $B$ is a generating transcendency basis, then $B_{Z}=B \cup B_{Y}$ satisfies the required conditions.

Every totally ordered subset $\left\{\left(Y_{i}, B_{Y_{i}}\right) \mid i \in J\right\} \subset \mathscr{L}$ has an upper bound, namely $\left(X_{J}=\cup Y_{i}, B_{J}=\cup B_{Y_{i}}\right)$. Indeed, if $B_{J}$ is not algebraically free over $R_{0}$, then there exists a finite subset $P \subset B_{J}$ which is not algebraically free. $P$ is contained in some $B_{j}$, which is algebraically free, so we have a contradiction. On the other hand, $B_{J} \subset R\left(X_{J}\right)_{0}^{\left(\omega\left(X_{J}\right)\right)}$ and, for each $Y_{i}$, the generating transcendency basis $B_{i}$ of $R\left(Y_{i}\right)_{0}^{\omega_{\mid Y_{i}}} / R_{0}$ is included in $B_{J}$, hence $R\left(Y_{i}\right)_{0}^{\omega_{\mid Y_{i}}} \subset R_{0}\left(B_{J}\right)$ and 
$R\left(X_{J}\right)_{0}^{\left(\operatorname{gr}\left(X_{J}\right)\right)} \subset R_{0}\left(B_{J}\right)$. So, $\left(X_{J}=\cup Y_{i}, B_{J}=\cup B_{Y_{i}}\right) \in \mathscr{L}$ and is bigger than all the $\left(Y_{i}, B_{Y_{i}}\right)$. It then follows from Zorn's lemma that there exists a maximal element $\left(X_{m}, B_{X_{m}}\right) \in \mathscr{L}$. We show that $X_{m}=X$.

Assume that there exists $x \in X \backslash X_{m}$ and let $Y=X_{m} \cup\{x\}$. Then $R(Y)_{0}^{\omega_{\mid Y}} / R\left(X_{m}\right)_{0}^{\omega_{\mid X m}}$ is purely transcendental of degree one with generating transcendency base $\{x\}$. Let $\alpha$ be the order of $\operatorname{deg}(x)$ over $\Gamma_{R}\left(\omega\left(X_{m}\right)\right)$ and take $z \in R\left(X_{m}\right)_{0}^{\omega_{\mid X m}}$ such that $\operatorname{deg}(z)=\alpha \operatorname{deg}(x)$. Then

$$
R(Y)_{0}^{\omega_{\mid Y}}=R\left(X_{m}\right)_{0}^{\omega_{\mid X m}}\left(z^{-1} x^{\alpha}\right),
$$

where $z^{-1} x^{\alpha}$ is transcendental over $R\left(X_{m}\right)_{0}^{\omega_{\mid X m}}$, and $R(Y)_{0}^{\omega_{\mid Y}} / R\left(X_{m}\right)_{0}$ is purely transcendental. This implies that $\left(X_{m}, B_{X_{m}}\right)$ is not maximal in $\mathscr{L}$, a contradiction. We conclude that $R(X)_{0}^{(\omega(X))} / R$ is purely transcendental and we have a generating transcendency basis $B_{X}$ indexed by $X$, so $\left[\left(R(X)^{\omega}\right)_{0}: R_{0}\right]_{t}=\#(X)=$ $\left[R(X)^{\omega}: R\right]_{t}$ by Proposition 5.1.

As an immediate consequence of Propositions 5.1 and 5.2, we have the following corollary.

COROLLARY 5.3. If $S / R$ is a purely gr-transcendental extension of $\Gamma$-graded fields of divisible type, then $S_{0} / R_{0}$ is purely transcendental and $[S: R]_{t}=\left[S_{0}\right.$ : $\left.R_{0}\right]_{t}$.

\section{Application to valued extensions}

6.1. The associated graded field. Let $(F, v)$ be a valued field with valuation group $\Gamma_{F}$ and let $\bar{F}$ be the residue field. For $\lambda \in \Gamma_{F}$, we have that $F_{\lambda}=\{x \in F \mid$ $v(x) \geq \lambda\}$ is a subgroup of $(F,+)$ and $F_{\lambda^{+}}=\{x \in K \mid v(x)>\lambda\}$ is a subgroup of $F_{\lambda}$. We also write $\operatorname{gr}(F)_{\lambda}=F_{\lambda} / F_{\lambda^{+}}$. In particular, $\operatorname{gr}(F)_{0}=\bar{F}$. On

$$
\operatorname{gr}(F)=\bigoplus_{\Gamma_{F}} \operatorname{gr}(F)_{\lambda},
$$

we define a multiplication as follows:

$$
\left(a+F_{\lambda^{+}}\right)\left(b+F_{\delta^{+}}\right)=\left(a b+F_{(\lambda+\delta)^{+}}\right)
$$

for $a \in \operatorname{gr}(F)_{\lambda}$ and $b \in \operatorname{gr}(F)_{\delta}$. This multiplication extends linearly to $\operatorname{gr}(F)$ and makes $\operatorname{gr}(F)$ into a $\Gamma_{F}$-graded field, called the associated graded field.

Let $\pi_{\lambda}: F_{\lambda} \rightarrow \operatorname{gr}(F)_{\lambda}$ be the canonical projection. For every $x \in F$, we put $\tilde{x}=\pi_{v(x)}(x)$. The group $\Gamma_{F}$ is totally ordered, and therefore torsion-free. Notice that if $E / F$ is an extension of valued fields, then $\operatorname{gr}(E) / \operatorname{gr}(F)$ is an extension of graded fields. More details on the associated graded field (or division ring) can be found in $[2,3,6,7,8,11]$. 
For an extension of valued fields $E / F$, we now define the following notions:

(1) $[E: F]_{t \cdot g}=[\operatorname{gr}(E): \operatorname{gr}(F)]_{t}$, the gradual transcendence degree of $E / F$;

(2) $[E: F]_{t \cdot r}=[\bar{E}: \bar{F}]_{t}$, the residual transcendence degree of $E / F$;

(3) $[E: F]_{t \cdot v}=\operatorname{rank}\left(\Gamma_{E} / \Gamma_{F}\right)$, the valuative transcendence degree of $E / F$.

We call $E$ a gradually (resp., residually, resp., valuatively) transcendental valued extension of $F$ if $\operatorname{gr}(E) / \operatorname{gr}(F)$ is a gr-transcendental graded field extension (resp., if $\bar{E} / \bar{F}$ is a transcendental field extension, resp., if $\Gamma_{E} / \Gamma_{F}$ is free).

If $T \subset E$ is such that $\tilde{T}=\{\tilde{t} \mid t \in T\}$ is gr-algebraically free over $\operatorname{gr}(E)$ (resp., a gr-transcendency basis of $\operatorname{gr}(E) / \operatorname{gr}(F)$ ), then we call $T$ gradually algebraically free (resp., a gradually transcendental basis of $E / F$ ).

Observe that if $T$ is a gradually transcendental basis of $E / F$, then there exists $T_{1} \subset E$ such that $T \cup T_{1}$ is a transcendency basis of $E / F$. Moreover, if $[E: F]_{t}$ is finite, then the cardinality of $T_{1}$ is independent of the choice of $T$. Indeed, we can take for $T_{1}$ a transcendency basis of $E / F(T)$, and if $[E: F]_{t}$ is finite, then the cardinality of $T_{1}$ is nothing but the transcendency degree of $E / F(T)$.

COROLLARY 6.1. Let $E / F$ be an extension of valued fields. Then

(1) $[E: F]_{t \cdot g} \leq[E: F]_{t}$ and $[E: F]_{t \cdot g}=[E: F]_{t \cdot r}+[E: F]_{t \cdot v}$;

(2) $\operatorname{rank}\left(\Gamma_{E} / \Gamma_{F}\right) \leq[E: F]_{t}$ and $[\bar{E}: \bar{F}]_{t} \leq[E: F]_{t}$;

(3) if $E / F$ is gradually purely transcendental (i.e., $\operatorname{gr}(E) / \operatorname{gr}(F)$ is purely grtranscendental) and $\Gamma_{E} / \Gamma_{F}$ is torsion $\left([E: F]_{t \cdot v}=0\right)$, then $E / F$ is residually purely transcendental.

We call a valued field extension $E / F$ gr-defective if $[E: F]_{t \cdot g}<[E: F]_{t}$, and non-gr-defective if $[E: F]_{t \cdot g}=[E: F]_{t}$. The extension $E / F$ is non-gr-defective if and only if there exists a transcendency basis $T$ of $E$ such that $\tilde{T}$ is a grtranscendency basis of $\operatorname{gr}(E) / \operatorname{gr}(F)$.

\section{REFERENCES}

[1] S. A. Amitsur, L. H. Rowen, and J.-P. Tignol, Division algebras of degree 4 and 8 with involution, Israel J. Math. 33 (1979), no. 2, 133-148.

[2] M. Boulagouaz, The graded and tame extensions, Commutative Ring Theory (Fes, 1992) (P.-J. Cahen, D. L. Costa, M. Fontana, and S.-E. Kabbaj, eds.), Lecture Notes in Pure and Appl. Math., vol. 153, Marcel Dekker, New York, 1994, pp. 27-40.

[3] __ Le gradué d'une algèbre à division valuée [The associated graded ring of a valued division algebra], Comm. Algebra 23 (1995), no. 11, 4275-4300 (French).

[4] - Algèbre à division graduée centrale [Central graded division algebra], Comm. Algebra 26 (1998), no. 9, 2933-2947 (French).

[5] __ Une généralisation du lemme de Hensel [A generalization of Hensel's lemma], Bull. Belg. Math. Soc. Simon Stevin 5 (1998), no. 5, 665-673 (French).

[6] __ An introduction to the Galois theory for graded fields, Algebra and Number Theory (Fez) (M. Boulagouaz and J.-P. Tignol, eds.), Lecture Notes in Pure and Appl. Math., vol. 208, Marcel Dekker, New York, 2000, pp. 21-31. 
[7] Y.-S. Hwang and A. R. Wadsworth, Algebraic extensions of graded and valued fields, Comm. Algebra 27 (1999), no. 2, 821-840.

[8] _ Correspondences between valued division algebras and graded division algebras, J. Algebra 220 (1999), no. 1, 73-114.

[9] H. Li and F. Van Oystaeyen, Zariskian Filtrations, $K$-Monographs in Mathematics, vol. 2, Kluwer Academic, Dordrecht, 1996.

[10] C. Năstăsescu and F. Van Oystaeyen, Graded Ring Theory, North-Holland Mathematical Library, vol. 28, North-Holland Publishing, Amsterdam, 1982.

[11] J.-P. Tignol, Algèbres à division et extensions de corps sauvagement ramifiées de degré premier [Wildly ramified division algebras and field extensions of prime degree], J. reine angew. Math. 404 (1990), 1-38 (French).

[12] J.-P. Tignol and A. R. Wadsworth, Totally ramified valuations on finite-dimensional division algebras, Trans. Amer. Math. Soc. 302 (1987), no. 1, 223-250.

[13] J. Van Geel and F. Van Oystaeyen, About graded fields, Nederl. Akad. Wetensch. Indag. Math. 43 (1981), no. 3, 273-286.

M. Boulagouaz: Département de Mathématiques, Faculté de Sciences et Techniques de Fès, University Sidi Mohammed ben Abdellah, BP 2202 Fès, Morocco

E-mail address: bou 1ag@caramai 1 .com; bou 1 ag@arocketmai 1 .com 


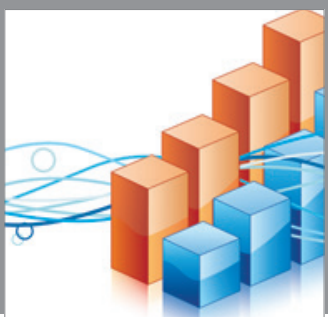

Advances in

Operations Research

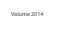

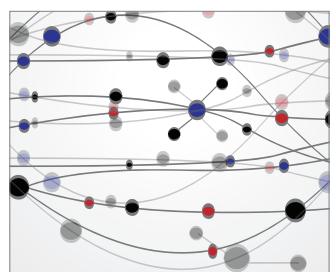

\section{The Scientific} World Journal
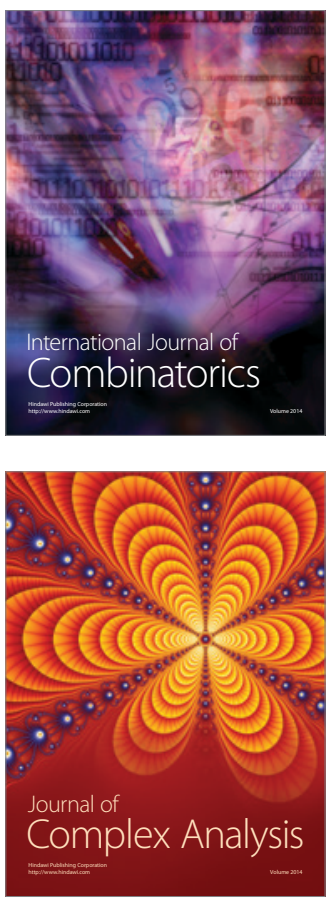

International Journal of

Mathematics and

Mathematical

Sciences
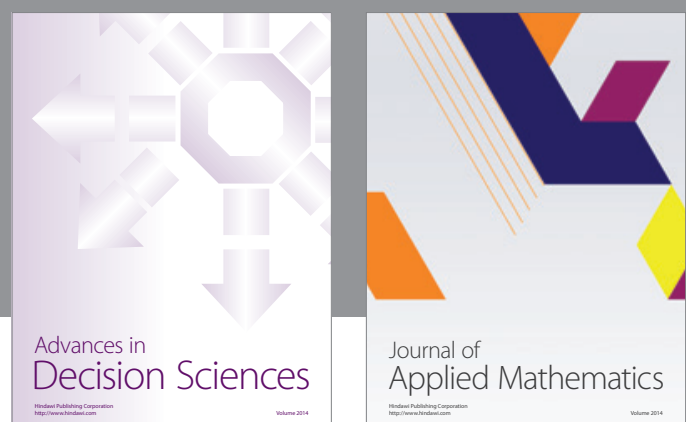

Journal of

Applied Mathematics
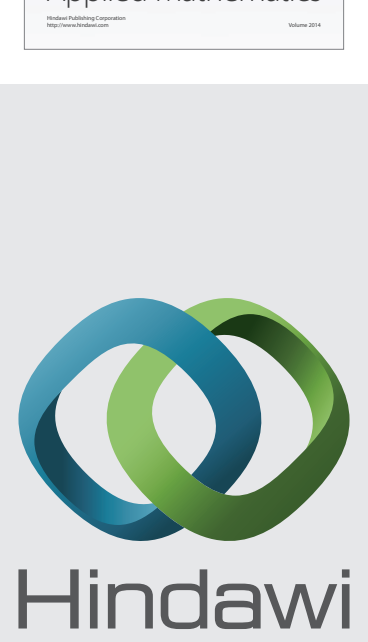

Submit your manuscripts at http://www.hindawi.com
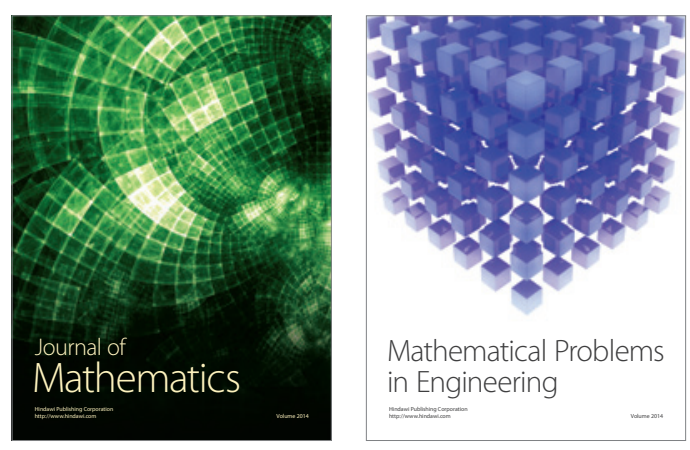

Mathematical Problems in Engineering
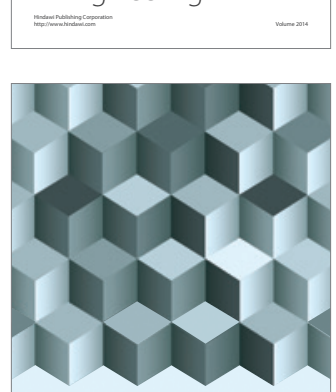

Journal of

Function Spaces
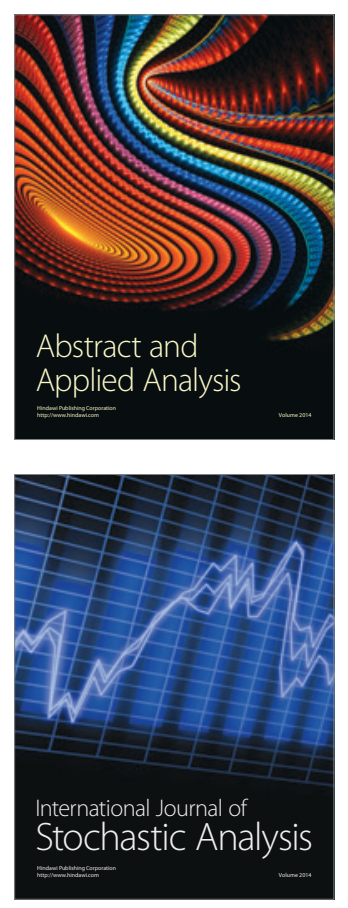

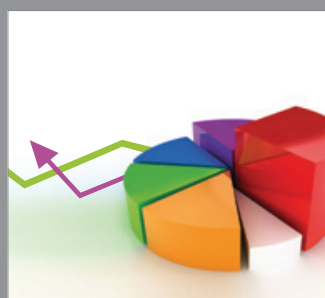

ournal of

Probability and Statistics

Promensencen
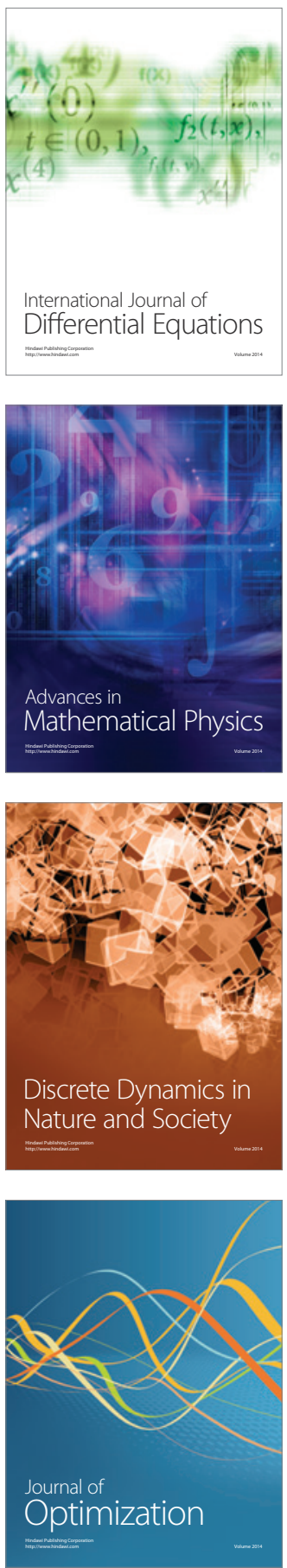